\title{
TAGUNG
}

\section{Deutsch-französische Führung in der erweiterten Union ohne Alternative?}

\author{
Gesa-Stefanie Brincker, Matti Roscher und Andrea Schilling*
}

Politische Führung bedeutet Verantwortung zu übernehmen, die Prioritäten der politischen Agenda zu definieren und Lösungshorizonte für ihre Umsetzung zu offerieren. Dass Führung in der erweiterten EU-27 in Anbetracht einer komplexeren Interessenlage einen deutlichen Bedeutungszuwachs erfährt, liegt auf der Hand. Zugleich taucht der Begriff ,leadership ' in den Verträgen jedoch nicht auf. War es in der Vergangenheit das deutsch-französische Tandem, das als ,Motor' den Integrationsprozess voranbrachte und nachhaltig prägte, ist die Zukunft politischer Führung in der Union offen. Während es kleinen Mitgliedstaaten an den nötigen Ressourcen mangelt, gerät das europapolitische Engagement der großen schnell in den Verdacht der Dominanz. Ungeachtet dieses Widerspruchs besteht ein klarer Bedarf an politischer Führung. In Anbetracht dessen verwundere es, wie stiefmütterlich die Forschung diese zentrale Thematik bislang behandelt habe, betonte der Vorsitzende des Wissenschaftlichen Direktoriums Professor Michael Kreile in seiner Einführung in die Thematik der interdisziplinären Jahresexpertenkonferenz des Instituts für Europäische Politik in Zusammenarbeit mit seinem Wissenschaftlichen Direktorium im September 2007. Die Fachtagung widmete sich dem Stand der deutsch-französischen Beziehungen und suchte nach Antworten auf die Frage nach der Zukunft politischer Führung in Europa (siehe Programmübersicht). $\mathrm{Zu}$ den Referentinnen und Referenten zählten Vertreter der Bundesregierung, Mitglieder des Wissenschaftlichen Direktoriums des IEP sowie weitere Frankreichexperten.

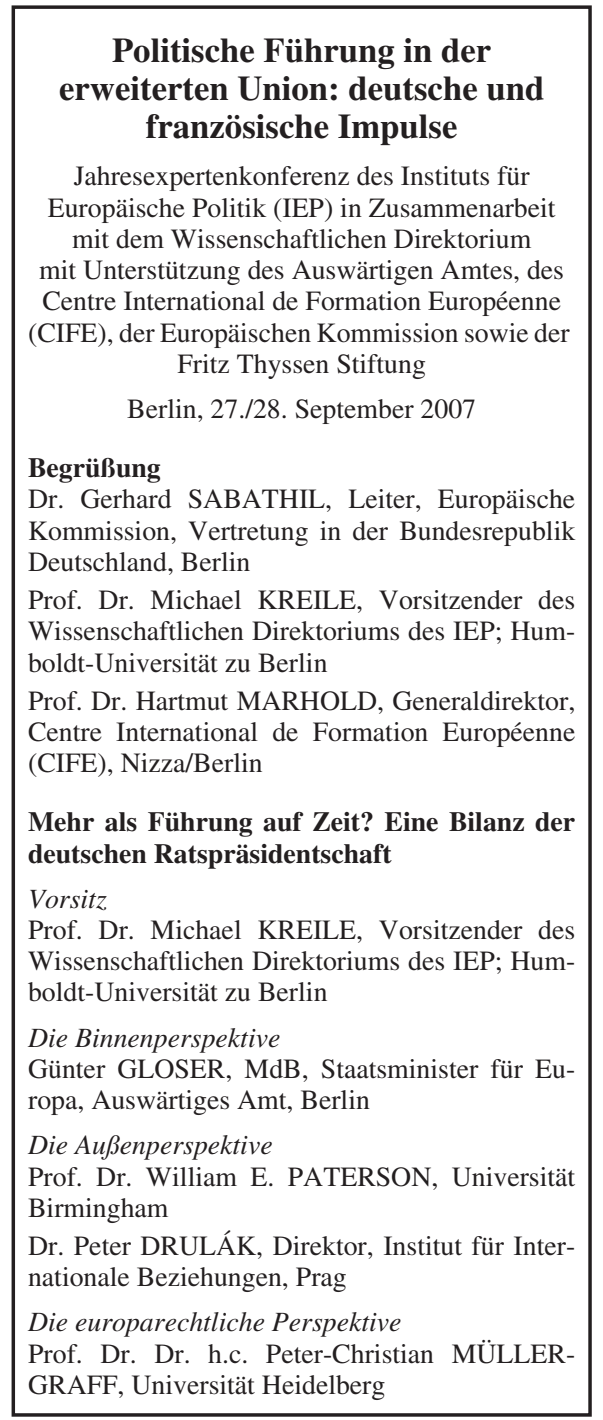

\section{Jahresexpertenkonferenz des Instituts für} Europäische Politik (IEP) in Zusammenarbeit mit dem Wissenschaftlichen Direktorium mit Unterstützung des Auswärtigen Amtes, des Centre International de Formation Européenne Fritz Thyssen Stiftung

Berlin, 27./28. September 2007

\section{Begrüßung}

Kommission, Vertre Deutschland, Berlin

Prof. Dr. Michael KREILE, Vorsitzender des Wissenschaftlichen Direktoriums des IEP; Hum-

Centre International de Formation Européenne Mehr als Führung auf Zeit? Eine Bilanz der deutschen Ratspräsidentschaft

Prof. Dr. Michael KREILE, Vorsitzender de Wissenschaftlichen Direktoriums des IEP; Humboldt-Universität zu Berlin

\section{Die Binnenperspektive} ropa, Auswärtiges Amt, Berlin

Die Außenperspektive

Prof. Dr. William E. PATERSON, Universität Birmingham

nationale Beziehungen, Prag

Die europarechtliche Perspektive

GRAFF, Universität Heidelberg

Gesa-Stefanie Brincker, M.E.S., Institut für Europäische Politik, Berlin. Matti Roscher, Institut für Europäische Politik, Berlin. Andrea Schilling, Institut für Europäische Politik, Berlin. 
Positive Bilanz der deutschen EU-Ratspräsidentschaft

Den Einstieg in die Tagung bildete die Erörterung der Ergebnisse der deutschen EU-Ratspräsidentschaft. Aus der Binnenperspektive fiel die Bilanz durchweg positiv aus und ergab folgendes Bild: Die Besonderheit der deutschen Verhandlungsleistung lag in dem eher vermittelnden Interessenausgleich zwischen den Mitgliedstaaten. Um selbst extrem gegensätzliche Partner zusammenzubringen, wurde gezielt nach bilateralen Lösungen gesucht. Die Präsidentschaft moderierte zwischen den unterschiedlichen Präferenzen und wahrte während des gesamten Prozesses eine transparente Vorgehensweise gegenüber den Verhandlungspartnern. Auf der Arbeitsebene konzentrierte sich die deutsche Ratspräsidentschaft auf die ,focal points' und versuchte das Ziel einer grundlegenden vertraglichen $\mathrm{Er}$ neuerung der Europäischen Union in einem Prozess steigender Verbindlichkeit zu erreichen. Die politische Steuerung durch den Ratsvorsitz erfolgte in engster Zusammenarbeit mit den EU-Institutionen. Von herausragender Bedeutung war dabei der Juristische Dienst des Ratssekretariats, der die Präsidentschaft - bedingt durch seine Mittlerrolle vom Verdacht der Dominanz entlastete. Im Ergebnis lag ein sehr konkretes Mandat für den Reformvertrag vor, das keine neuen Verhandlungen zuließ und zugleich die Substanz des Verfassungsvertrages erhielt.

Auch die Referenten aus dem Vereinigten Königreich sowie der Tschechischen Republik bestätigten aus der Außenperspektive die positive Einschätzung der deutschen Ratspräsidentschaft. Sowohl der Führungsstil während der Präsidentschaft als auch die Ergebnisse fanden breite Zustimmung. Ausschlaggebend waren nicht zuletzt auch die günstigen Rahmenbedingungen: Während in den anderen großen EU-Mitgliedstaaten Frankreich und dem Vereinigten Königreich kurz zuvor ein neuer Präsident beziehungsweise Regierungschef die Amtsgeschäfte übernommen hatte, zeichnete sich die deut-
Programmatik und Perspektiven französischer Europapolitik nach den Wahlen

Vorsitz

Prof. Dr. Hartmut MARHOLD, Generaldirektor, Centre International de Formation Européenne (CIFE), Nizza/Berlin

Innenpolitische Voraussetzungen französischer Europapolitik

Dr. Sylvie GOULARD, Präsidentin der Europäischen Bewegung Frankreich, Paris

Prof. Dr. Joachim SCHILD, Universität Trier

Eine neue französische Europapolitik unter Sarkozy?

Prof. Dr. Anne-Marie LE GLOANNEC, Centre d'Études et de Recherches Internationales, Paris

Inhalte und Weg zur Wiederbelebung des deutsch-französischen Führungsduos

Dr. Daniela SCHWARZER, Stiftung Wissenschaft und Politik, Berlin

PD Dr. Matthias WAECHTER, Direktor des Institut Européen des Hautes Études Internationales (IEHEI), Centre International de Formation Européenne (CIFE), Nizza

Deutsche und französische Impulse auf wichtigen Politikfeldern

Vorsitz

Dr. Jürgen TRUMPF, Generalsekretär a.D. des Rates der Europäischen Union; Präsident des IEP, Bonn

Die Überprüfung des Haushalts 2008/09

Dr. Friedrich HEINEMANN, Zentrum für Europäische Wirtschaftsforschung, Mannheim

Dr. Christian WEISE, Europäische Kommission, Brüssel

Zielrichtung und Prioritäten der Nachbarschaftspolitik (Osteuropa vs. Union Méditerranéenne?)

Prof. Dr. François BAFOIL, Centre National de la Recherche Scientifique / Centre d'Études et de Recherches Internationales, Paris

Dr. Barbara LIPPERT, Stellv. Direktorin, Institut für Europäische Politik, Berlin 
sche Bundesregierung durch politische Stabilität sowie parteiübergreifende Einigkeit hinsichtlich der europapolitischen Prioritäten aus. Bundeskanzlerin Merkel profitierte von dem Vertrauensvorschuss, der ihr auf europäischer Ebene entgegengebracht wurde, und führte die Europäische Union, trotz der im Vorfeld hohen Erwartungen, erfolgreich aus der Verfassungskrise. Obwohl Konfliktschlichtung erfahrungsgemäß oft von den Präsidentschaften ,kleinerer' EU-Mitgliedstaaten erzielt wird, war es dieses Mal der Regierung des größten Mitgliedstaates gelungen, wirksam zwischen den divergierenden Interessen seiner EU-Partner zu vermitteln und dabei zugleich eigene Interessen (wie den Erhalt der inhaltlichen Substanz des Verfassungsvertrages) umfassend zu wahren. Für diese Zielsetzung stand der Bundesregierung nicht zuletzt ein Großteil der 17 Mitgliedstaaten, die bereits den Verfassungsvertrag ratifiziert hatten, unterstützend zur Seite.

Inzwischen haben die Staats- und Regierungschefs im Oktober 2007 auf der Grundlage des Mandats den Lissabonner Vertrag ausgehandelt, der daraufhin am 13. Dezember 2007 feierlich unterzeichnet wurde. Die Ratifikation des Lissabonner Vertrags durch die Mitgliedstaaten steht im Jahr 2008 an. Während einige Konferenzteilnehmer auf mögliche Stolpersteine und Unsicherheiten im Ratifizierungsprozess hinwiesen, wurde von Seiten der Bundesregierung bekräftigt, dass es keinen „Plan B“ gebe.

\section{Chancen deutsch-französischer Kooperation}

Für den Erfolg der Vertragsverhandlungen war neben der deutschen Führungsstärke insbesondere die Rolle Frankreichs ausschlaggebend. Mit seinem Konzept des traité simplifié trug der neu gewählte französische Präsident Nicolas Sarkozy zur Überwindung der Verfassungskrise der Union bei. Die Analyse der französischen Innen- und Europapolitik bildete den zweiten Schwerpunkt der Konferenz. Aus Sicht der Frankreichexperten zeichnen sich folgende Trends ab: Durch den Regie-
Politische Führung durch Kernstaaten oder durch Institutionen?

Vorsitz

Prof. Dr. Heinrich SCHNEIDER, Universität Wien; Ehrenvorsitzender des Wissenschaftlichen Direktoriums des IEP, Berlin

\section{Einführungen}

Dirk LÖLKE VLR, Leiter des Referats 511, EU-Grundsatzangelegenheiten, Bundeskanzleramt, Berlin

Prof. Dr. Wolfgang WESSELS, Universität zu Köln

Kommentar

Dr. Andreas MAURER, Stiftung Wissenschaft und Politik, Berlin

\section{Schlussfolgerungen}

Prof. Dr. Michael KREILE, Vorsitzender des Wissenschaftlichen Direktoriums des IEP; Humboldt-Universität zu Berlin

Prof. Dr. Mathias JOPP, Direktor, Institut für Europäische Politik, Berlin

rungswechsel im Mai 2007 hat sich Frankreichs Engagement in und für Europa deutlich dynamisiert. Hatte sich das Land unter Präsident Chirac zuletzt kaum noch auf der europäischen Bühne gezeigt, ist es unter Sarkozy wieder präsent. Gleichwohl unterliegt seine Europapolitik innenpolitischen Handlungsrestriktionen. Sarkozys eigenem Anspruch nach soll seine Europapolitik für die Wähler mehrheitsfähig sein. Es gilt der Primat der Innenpolitik. Europa- und Außenpolitik sind folglich nur Funktionen innenpolitischer Imperative. Das französische Dilemma ist durch eine Ambivalenz zwischen nationaler Souveränität und verstärktem europapolitischen Engagement gekennzeichnet. Sarkozy muss versuchen den außenpolitischen Ansprüchen gerecht zu werden und dabei gleichzeitig innenpolitische Empfindlichkeiten berücksichtigen. Besonders Wirtschafts- und Sozialpolitik sind innenpolitisch sensibel und hier verfolgt Frankreich seine eigenen Interessen mit Nachdruck. Die Europäische Union 
dürfe nicht das Trojanische Pferd der Globalisierung werden, stellte der neugewählte Präsident gleich zu Beginn klar. Die großen Projekte für Europa fehlten und überhaupt sei Sarkozy kein überzeugter Europäer, bemerkte eine Referentin.

Neben dieser unter den Referenten mehrheitlich geteilten Einschätzung der französischen Europapolitik wurde jedoch auch eine weitere Argumentationslinie deutlich. Hierbei wurde die starke Machtposition des Präsidenten und sein Wille zum Tabubruch betont: Sarkozy hat die Ressourcen und den Willen, der Europapolitik seines Landes ein eigenständiges Profil zu geben. Das Bemühen um Europa ist glaubwürdig. Alles in allem bestand unter den Referenten Konsens, dass eindeutige Schlussfolgerungen über den künftigen europapolitischen Kurs Frankreichs verfrüht seien. Unter der Ratspräsidentschaft ab Juni 2008 werde sich klarer zeigen, welches Europa Frankreich wünscht.

Im Hinblick auf konkrete Politikfelder für die deutsch-französische Kooperation kamen aus Sicht der Referenten vorrangig die Europäische Nachbarschaftspolitik (ENP), Teile der Europäischen Sicherheits- und Verteidigungspolitik (ESVP) und die europäische Finanzregulierung infrage. Ob Fortschritte ohne das Vereinigte Königreich im Bereich der ESVP erzielt werden können, blieb unter den Konferenzteilnehmern bis zuletzt umstritten.

Auch die Bewertung der ENP aus beiden Länderperspektiven fiel kontrovers aus. Zwar bestand Einigkeit darin, dass die politischen Eliten beider Länder eine Europäisierung der Nachbarstaaten auch ohne Beitrittsversprechen und somit eine starke ENP als Alternative zu weiteren Beitritten wünschten. Allerdings zeigten sich bereits bei grundlegenden Fragen der geografischen Prioritätensetzung zwischen Süden und Osten Divergenzen. Während das französische Konzept einer Mittelmeerunion noch recht ,nebulös ' und für deutsche Akteure weniger überzeugend sei, fehlten im Gegenzug vergleichbare deutsche
Projekte, welche die östliche EU-Nachbarschaft einbeziehen. Um den geografischen Gegensatz auszubalancieren und die deutschfranzösische Kooperation im Rahmen der ENP zu fördern, gäbe es die Möglichkeit die Rollen zu vertauschen. So könnte sich Frankreich, einem Vorschlag des Podiums zufolge, für die Interessen der östlichen Nachbarstaaten einsetzen, Deutschland demgegenüber für die der südlichen. Aus dem französischen Blickwinkel sei die Erweiterung der Union zum Ende gekommen, eine Phase der institutionellen Konsolidierung nun notwendig, erläuterte ein Referent. Die Mittelmeerunion wäre geeignet, die Türkei in die euromediterrane Strategie einzubeziehen, um ihr die Beitrittsperspektive unmissverständlich zu nehmen. Mit einer gemeinsamen deutschfranzösischen Initiative zur Frage des Türkeibeitritts sei somit nicht zu rechnen.

Zentrale Bewährungsproben für die deutschfranzösische Kooperation sahen einige Referenten in der Budgetreform und insbesondere in der Reform der Gemeinsamen Agrarpolitik (GAP) sowie einer gemeinschaftlichen Energiepolitik. Gerade im Bereich der GAP seien die Hoffnungen auf eine Modernisierung der Strukturen gering, denn obschon Frankreichs Interesse an den Subventionszahlungen für heimische Landwirte sinke, bleibe sein Standpunkt unklar und die Frage, ob es den Status quo erhalten wolle, unbeantwortet. In diesem Fall könnte Polen, aber auch einige andere neue Mitgliedstaaten, zu einem Verbündeten Frankreichs zur Verteidigung der GAP-Zahlungen werden.

Obwohl Deutschland und Frankreich weiterhin ein breites Spektrum unterschiedlicher Interessen repräsentieren, wodurch deutschfranzösische Kompromisse auch in der groBen EU-27 am ehesten konsensfähig sein könnten, bleiben die Erfolgsaussichten deutsch-französischer Führungsimpulse angesichts der zum Teil divergenten Leitbilder in der erweiterten Europäischen Union unsicher. 
Ausblick auf die institutionelle Zukunft der Europäischen Union

Den abschließenden Schwerpunkt der Konferenz bildete der Ausblick auf die Auswirkungen der Primärrechtsreform, welche die gemeinschaftlichen Institutionen und ihre Handlungsweisen grundlegend erneuert. Im Zentrum der Diskussion standen dabei die Fragen nach der operativen Rolle des Präsidenten des Europäischen Rates und nach den Konsequenzen der im Vertrag von Lissabon vorgesehenen und bereits praktizierten Triopräsidentschaften. Dabei wurden zwei konträre Argumente besonders deutlich: Einerseits könnten beide Neuerungen als positiver Schritt zu größerer Kontinuität interpretiert werden. Andererseits könnte diese Kontinuität aber auch politische Impulse seitens der nationalen Regierungen erschweren sowie ,Ad-hoc'-Koalitionen zur Durchsetzung von Interessen verhindern. Kontrovers diskutiert wurde des Weiteren der Gegensatz zwischen der mit dem Vertrag von Lissabon beabsichtigten Stärkung der Institutionen und der verstärkten Zusammenarbeit. Während eine solche Avantgarde Integrationsimpulse geben kann, besteht jedoch gleichzeitig die Gefahr, die europäischen Institutionen langfristig auszuhöhlen, solange die Zusammenarbeit dauerhaft auf einzelne Mitgliedstaaten beschränkt bleibt. Die Konferenzteilnehmer betonten, wie wichtig es im Kontext jedweder Kooperation sei, dass die großen Mitgliedstaaten die Interessen der kleinen berücksichtigen und diese nicht marginalisieren.

Unbestritten war die große Bedeutung deutsch-französischer Impulse für die EU-27. Zwar werde die Arbeit des Tandems in der erweiterten Union schwieriger und die Erfolgschancen unsicherer, letztendlich bleibe sie aber alternativlos, da von keinem der anderen großen Mitgliedstaaten entsprechende Impulse erwartet werden könnten. Insbesondere in schwierigen Situationen, wenn die nationalen Interessen der Mitgliedstaaten weit auseinander lägen, sei die Zusammenarbeit zwischen Paris und Berlin für eine Kompromissfindung nach wie vor maßgeblich. Dies gelte beispielsweise für EU-Erweiterungen oder Vertragsänderungen. Die Kommunikation zwischen den Institutionen beider Staaten sei jedoch noch ausbaufähig.

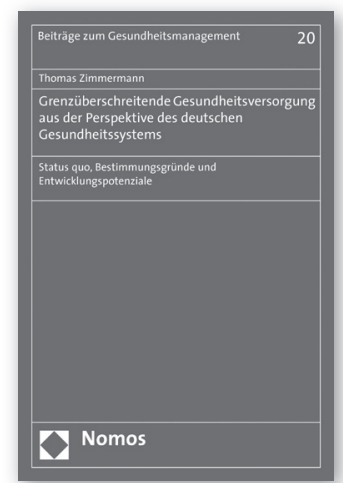

\section{Grenzüberschreitende Gesundheits- versorgung aus der Perspektive des deutschen Gesundheitssystems}

Status quo, Bestimmungsgründe und Entwicklungspotenziale

Von Thomas Zimmermann

2008, ca. 360 S., brosch., ca. 59,- $€$, ISBN 978-3-8329-3286-2

(Beiträge zum Gesundheitsmanagement, Bd. 20)

Erscheint März 2008 